\title{
El costo de la "remasterización" del Arbitraje
}

\author{
Álvaro Estrada Rosas \\ Abogado por la Universidad de Lima \\ Miembro Asociado de ADV Editores - Revista ADVOCATUS.
}

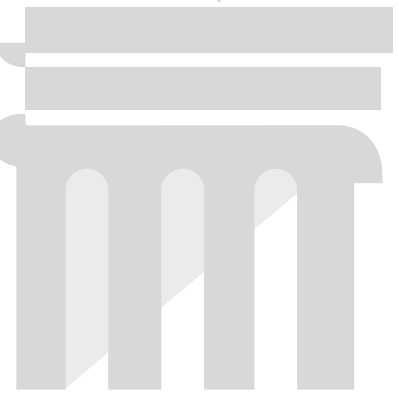

SUMARIO:

I. Introducción.

II. Breve análisis de la evolución del arbitraje.

III. El costo de "remasterizar" el arbitraje: algunas cuestiones asociadas al uso de tecnologías en el arbitraje.

1. ¿Se debe pactar necesariamente el uso de las tecnologías en el convenio arbitral para que las actuaciones sean virtuales?

2. ¿Qué sucede si las partes convienen el uso de las tecnologías y pactan una tecnología en específico para el arbitraje, 0 deciden acudir a una institución arbitral determinada y someterse a las herramientas tecnológicas que esta exige para sus procesos, pero luego esta tecnología es prohibida como política interna de una de las partes?

3. ¿Es más eficiente, en términos de costo y tiempo, establecer tecnologías para el arbitraje? Y, de ser así, ¿es más eficiente establecer las tecnologías a usar en el arbitraje antes o luego de iniciado el proceso?

4. ¿Si se presentan problemas en el uso de las tecnologías, podrá dispensarse a las partes de utilizarlas?

5. ¿El tribunal arbitral puede establecer sanciones relacionadas a las actuaciones de las partes y el uso de tecnologías?

6. Al momento de designar al árbitro, ¿la parte deberá tomar en cuenta si éste se encuentra familiarizado con el uso de las tecnologías pactadas?

7. ¿Se ha generado un "nuevo derecho" de las partes en el arbitraje de "testear" la conectividad, audio y video antes de las audiencias? ¿Qué sucede si se le niega esta solicitud a una parte?

IV. Conclusión. 


\title{
RESUMEN:
}

A raíz de la pandemia producida por la COVID-19, el arbitraje se ha visto obligado a "remasterizarse", para lo cual ha necesitado implementar el uso de herramientas virtuales. No obstante, la implementación de la tecnología en el arbitraje genera cuestiones o situaciones sumamente problemáticas que son abordadas en el presente artículo de la mano de guías internacionales sobre el uso de la tecnología en el arbitraje.

Palabras clave: arbitraje, audiencia, internacional, tecnología, virtual, pandemia.

\begin{abstract}
:
As a result of the COVID-19 pandemic, arbitration has been forced to "remaster" itself, for which it has needed to implement the use of virtual tools. However, the implementation of technology in arbitration generates highly problematic issues or situations that are addressed in this article with the help of international guidelines on the use of technology in arbitration.

Keywords: arbitration, hearing, international, technology, virtual, pandemic.
\end{abstract}

\section{INTRODUCCIÓN}

Hace unos pocos años llegó la moda de la "remasterización". La remasterización consiste en mejorar - $\mathrm{o}$ actualizar - las calidades técnicas y estéticas de un producto que ya existe - 0 existió- en el mercado.

La remasterización ha aplicado en varios ámbitos: en la música, en el cine, en los videojuegos, etc. Generalmente se suele escoger un producto que, por la acogida que tuvo en su época, se convirtió en un clásico, y se remasteriza para redefinir sus calidades estéticas usando las nuevas técnicas tecnológicas.

Un ejemplo podría ser el del videojuego "Crash Bandicoot", que se lanzó en 1996 y que, a pesar de tener una historia bastante simple', tuvo mucha acogida en su momento. La remasterización de este videojuego fue lanzada en 2017, obteniendo ventas muy elevadas.

Así como este videojuego, y otros muchos ejemplos más, el arbitraje también ha sufrido una remasterización. Efectivamente, el arbitraje ha necesitado redefinirse usando las nuevas tecnologías.
Si bien en los últimos años el arbitraje ha ido incorporando figuras e instituciones jurídicas que han permitido que se consolide como mecanismo de solución de controversias, la remasterización del arbitraje se ha producido con la implementación de la tecnología en los procesos arbitrales, que se volvió obligatoria para cada arbitraje como consecuencia de la pandemia de la COVID-19. Así, el uso de la tecnología en el arbitraje ha pasado de ser la excepción a la regla general.

Efectivamente, en el caso del Perú, a partir del 16 de marzo de 2020, entramos a un período de aislamiento obligatorio; y como consecuencia quedaron suspendidas todas las actividades presenciales, lo que produjo en un primer momento la suspensión de todos los plazos de los arbitrajes, y que progresivamente fueron reanudándose por la implementación de la tecnología.

Inevitablemente la incorporación de las tecnologías en el arbitraje - o que el arbitraje se haya remasterizado- ha supuesto un costo, porque se han generado situaciones y cuestiones que tarde o temprano van a surgir en la práctica diaria, por lo que en este trabajo se abordarán

1. La historia es de un marsupial que desea evitar los planes del malvado Doctor Neo Cortex de dominar el mundo y, en el camino intenta, rescatar a su novia Tawna. 
algunas ideas que deben conocerse de cara a esta nueva manera de arbitrar.

\section{BREVE ANÁLISIS DE LA EVOLUCIÓN DEL ARBITRAJE}

El arbitraje es un mecanismo de solución de controversias contractual, en donde las partes de una relación jurídica determinada convienen para que sus controversias sean excluidas del fuero judicial y sean resueltas por un tercero llamado árbitro-. Se decide acudir al arbitraje por los diversos beneficios que caracterizan al arbitraje, como la especialización, flexibilidad, celeridad, confidencialidad, entre otros. Todo ello lo hace un mecanismo de solución de controversias más eficiente.

El arbitraje en nuestro país ha sufrido cambios drásticos desde que se incorporó como mecanismo de solución de controversias. Ha ido transformándose pasando de ser un mecanismo de solución de controversias excesivamente formal a uno bastante flexible y efectivo ${ }^{2}$. Cabe precisar que recientemente se han incluido modificaciones a la Ley de Arbitraje. Primero, a través del Decreto Legislativo $1231^{3}$ para evitar fraudes en los procesos arbitrales, y luego, mediante el Decreto de Urgencia 020-20204, que introduce una regulación especial para los arbitrajes en los que el Estado interviene como parte.

La transformación del arbitraje no ha quedado ahí. Podríamos decir que, en estos últimos años, se han incorporado figuras novedosas al arbitraje debido a las buenas prácticas internacionales en esta materia, que lo dotan de mayor eficiencia y destacan frente al fuero ordinario.

En estos últimos años han aparecido figuras como la confirmación de árbitros, el arbitraje de emergencia, las reglas de la International Bar Association —en adelante, "Reglas de la IBA"一, las reglas de Praga sobre la tramitación eficiente de los procedimientos en el arbitraje internacional —en adelante, "Reglas de Praga"-, el arbitraje acelerado, entre otros.

La confirmación de árbitros, que ha sido incluida en las reglas de determinadas institucionales arbitrales ${ }^{5}$, permite a la institución arbitral realizar una suerte de filtro para determinar si un árbitro debe formar parte de los miembros del tribunal arbitral que resolverán un caso específico. El objetivo es que la institución que administra el proceso arbitral pueda cerciorarse que el árbitro designado cumple con ser imparcial e independiente, y que es adecuado para resolver el caso.

El arbitraje de emergencia, figura incorporada en los reglamentos de algunas instituciones arbitrales ${ }^{6}$, a diferencia de una medida cautelar judicial - que el juez, por la carga procesal, puede demorar en dictar-, tiene por finalidad que un árbitro - distinto al que resolverá la controversia de fondo- se constituya inmediatamente a fin de dictar una medida cautelar con

2. En un primer momento, el arbitraje estuvo regulado en el Código de Procedimientos Civiles de 1912, que incorporaba el "fuero arbitral"; y luego, estuvo en una sección del Código Civil de 1984, bajo las figuras de "cláusula compromisoria" y "compromiso arbitral". No fue sino hasta el 6 de enero de 1996, con la Ley General de Arbitraje —Ley 26572_, que el arbitraje estuvo regulado en un cuerpo normativo independiente. Finalmente, el Decreto Legislativo 1071 — Ley de Arbitraje — es el que actualmente se encuentra vigente.

3. Publicado el 26 de septiembre de 2015.

4. Publicado el 24 de enero de 2020.

5. Esta figura la encontramos en el Reglamento de Arbitraje de la Cámara de Comercio de Finlandia de 2013 y en el Reglamento de Arbitraje de la Cámara de Comercio de Lima de 2017.

6. Reglamento de Arbitraje de la Cámara de Comercio Internacional de París de 2017 y en el Reglamento de Arbitraje de la Cámara de Comercio de Lima de 2017. 
carácter de urgencia, solicitada por cualquiera de las partes, para que su derecho o reclamo no sea irreparable por el tiempo que demoraría un proceso determinado.

La International Bar Association decidió codificar la práctica recurrente internacional en materia de pruebas documentales y no documentales, de conflictos de intereses de quienes participan en un arbitraje y de representación de las partes. Así, nacen las denominadas Reglas de la IBA?

Las Reglas de Praga nacen, en contraposición a la lógica procedimental establecida por las Reglas de la IBA que permiten a las partes regular lo relacionado a las actuaciones arbitrales, para dotar al tribunal arbitral la facultad de regular el proceso de la manera más eficiente que considere, no siendo necesario en la mayoría de casos el consentimiento o acuerdo de las partes ${ }^{8}$.

El arbitraje acelerado es una figura que ha aparecido recientemente, en atención a las demandas de los usuarios del arbitraje relativas a que sus controversias no se resolvían de una manera rápida. Diversas instituciones arbitrales han incorporado esta figura en sus reglamentos ${ }^{9}$.

Vemos entonces que el arbitraje ha ido implementando, con el pasar de los años, mecanismos y/o figuras que lo hacen más llamativo, adecuándose no solo a las necesidades de los usuarios sino reafirmando que sirve como un mecanismo de solución de controversias eficiente.

\section{EL COSTO DE "REMASTERIZAR" EL ARBI- TRAJE: ALGUNAS CUESTIONES ASOCIA- DAS AL USO DE LAS TECNOLOGÍAS EN EL ARBITRAJE}

Con la entrada al siglo XXI y la masificación de la tecnología de la información, el arbitraje ha necesitado prestarse de las facilidades que proporcionan las herramientas virtuales para adaptarse a los tiempos actuales. En pocas palabras, ha necesitado remasterizarse.

Si bien en un inicio los usuarios del arbitraje abogados, árbitros, partes en litigio, secretarios arbitrales, etc.- implementaban las tecnologías como excepción para el desarrollo de las actuaciones arbitrales, con ocasión de la pandemia de la COVID-19, las herramientas y soportes virtuales se han convertido en una necesidad.

En esa línea, es importante precisar que en estos últimos años se ha publicado un sinfín de guías, nacionales e internacionales, que permiten que el arbitraje pueda adecuarse a las nuevas tecnologías de la información y que, con la ayuda de estas herramientas, puedan continuar desarrollándose las actuaciones arbitrales, pero ahora de manera virtual.

Una de estas guías es el denominado Protocolo de Seúl Sobre Videoconferencia en Arbitraje In-

7. Según el numeral 1 del Preámbulo, las Reglas de la IBA sobre la práctica de la prueba "buscan proporcionar un procedimiento eficiente, económico y equitativo para la práctica de prueba en arbitrajes internacionales, particularmente en aquellos que surgen entre Partes de distintas tradiciones jurídicas. Están diseñadas para complementar las disposiciones legales y las reglas institucionales, ad hoc u otras reglas que se apliquen al desarrollo del arbitraje."

8. El primer párrafo del Preámbulo de las Reglas de Praga sobre la tramitación señala que las Reglas de Praga: “(...) pretenden proporcionar a los tribunales arbitrales y a las partes unas pautas o sugerencias para incrementar la eficiencia del arbitraje, potenciando un papel más activo de los tribunales arbitrales en la tramitación de los procedimientos."

9. Solo por mencionar unos cuantos, esta figura ha sido incorporada en los reglamentos de las siguientes institucionales arbitrales: Reglamento de Arbitraje del Centro de Arbitraje de la Cámara de Comercio de Lima —2017—, Reglamento de Arbitraje del Centro de Análisis y Resolución de Conflictos de la Pontificia Universidad Católica del Perú —2017—, Reglamento para Arbitraje Acelerado de la Cámara de Comercio de Finlandia —2013 — y el Reglamento de la Cámara de Comercio Internacional de París -2017_. 
ternacional -en inglés, Seoul Protocol on Video Conferencing in International Arbitration ${ }^{10}$; en adelante, el "Protocolo de Seúl"—, que sugiere las mejores prácticas para el planeamiento, prueba y conducción de la video conferencia en los arbitrajes internacionales.

Otro documento importante es el Reporte de la Tecnología de la Información en el Arbitraje Internacional, emitido por la Cámara de Comercio Internacional -en inglés, Information Technology in International Arbitration; en adelante, el "Reporte $\mathrm{CCl}^{-}-$, que propone una serie de supuestos que pudieran presentarse en la realidad en el marco del uso de las tecnologías de la información, y que aconseja las mejores herramientas para la aplicación de las tecnologías en el arbitraje.

Existen muchos otros documentos que sirven como guía para las actuaciones arbitrales virtuales, que, de hecho, fueron publicadas para buscar una solución a la suspensión de las actuaciones arbitrales causada por las medidas de salubridad que impedían las actuaciones arbitrales presenciales ${ }^{11}$. No obstante, este trabajo se basará esencialmente en las dos últimas mencionadas, que cubren gran parte de las cuestiones que se presentan o pudieran presentarse en un proceso que se ayuda de las tecnologías de la información.

\section{1. ¿Se debe pactar necesariamente el uso de las tecnologías en el convenio arbitral}

para que las actuaciones sean virtuales? La primera pregunta que podría surgir es si las partes de un contrato deben pactar necesariamente el uso de las tecnologías para llevar a cabo sus arbitrajes. Es decir, si las partes no pactan un arbitraje con el uso de tecnologías, ¿el arbitraje deberá llevarse de manera presencial? Ello depende.

Sería ideal que se pacte el uso de las tecnologías y el tipo específico de tecnología que usarían las partes para llevar a cabo el arbitraje en el que se resolverán sus controversias. Como se verá más adelante, el acordar sobre el uso de las tecnologías y la tecnología en específico permite evitar que surja una controversia innecesaria en ese sentido.

Aunque remotamente, el problema que podría surgir es que las partes pacten un tipo de tecnología que, al momento de convenirla, era eficiente para ellas pero que, a la fecha de surgir las controversias, haya quedado desfasada y existan otras que la superen ${ }^{12}$. En ese escenario no quedaría más remedio que las partes acuerden someterse a esa nueva y mejor tecnología, porque de lo contrario tendrán que utilizar la que han pactado en su convenio arbitral.

Sin perjuicio de lo anterior, el panorama ideal sería que las partes hayan previsto someterse a un arbitraje de manera virtual identificando las herramientas tecnológicas a usar.

10. Diseñada por los abogados Kevin Kim, Yu-Jin Tay, Ing Loong Yang y Seung Ming Lee, con el apoyo de la Korean Commercial Arbitration Board-KCAB y la Seoul International Dispute Resolution Centre-SIDRC.

11. Solo por mencionar algunos centros de arbitrajes nacionales, tenemos que el Centro de Arbitraje de la Cámara de Comercio de Lima publicó en abril del año 2020 la Nota Práctica Nº1/2020 para garantizar la virtualización de las actuaciones arbitrales. Lo mismo sucedió con la publicación de la Guía de Arbitrajes Virtuales del Centro Internacional de Arbitraje de Amcham Perú en mayo del año 2020.

En el plano internacional, como se ha precisado en este trabajo, se han publicado una serie de guías y/o protocolos que brindan lineamientos para el uso de las tecnologías en el arbitraje. Una guía adicional, que intencionalmente no es desarrollada en este trabajo pero que igual resulta ser una de las más relevantes, es la Guía para el Uso del Videolink bajo la Convención de la Haya publicada en abril de 2020 —en inglés, Guide of Use of Video-Link under Evidence Convention-.

12. Cámara de Comercio Internacional, Reporte de Tecnologías de Información en el Arbitraje Internacional (Francia, 2017), 4. 
Ahora bien, ¿qué sucede si las partes han pactado acudir a arbitraje, pero no han pactado el uso de las tecnologías? ¿No se podrá llevar a cabo un arbitraje con el uso de herramientas virtuales? Y aquí, como todo en Derecho, depende.

Lo primero que podría señalarse es que, si las partes no han establecido en su convenio arbitral someterse a arbitraje con la ayuda de las tecnologías, y salvo que no haya un acuerdo posterior entre ellas que sí lo permita o alguna manifestación de las partes de ser notificadas por algún medio electrónico ${ }^{13}$, tendrá que llevarse exclusivamente de manera presencial. ${ }^{14}$

Otro escenario es si las partes no han pactado en su convenio arbitral utilizar las tecnologías, pero han sometido sus controversias a la administración de una institución de arbitraje determinada que, entre otras cosas, promueve el uso de las tecnologías.

Aquí las partes no podrían evitar llevar a cabo las actuaciones arbitrales con la ayuda de las herramientas tecnológicas.

Por ejemplo, el Reglamento de Arbitraje 2017 de la Cámara de Comercio Internacional establece que las notificaciones, entre otras, se podrán realizar de manera virtual ${ }^{15}$, y que el tribunal arbitral puede celebrar audiencias virtuales en las que no sea esencial la asistencia presencial de las partes ${ }^{16}$. Es decir, el reglamento permite realizar actuaciones arbitrales de manera virtual.

Por lo tanto, es conveniente pactar el uso de las tecnologías y la tecnología en específico que se usará para el arbitraje porque de lo contrario, va a depender mucho en qué circunstancia nos encontremos para determinar si el arbitraje se puede llevar de manera virtual o solo podrá desarrollarse presencialmente. Este último escenario solo se daría si no existe alguna manifestación de las partes que permita si quiera las notificaciones por algún medio electrónico.

\section{2. ¿Qué sucede si las partes convienen el uso de las tecnologías y pactan una tec- nología en específico para el arbitraje o deciden acudir a una institución arbitral determinada y someterse a las herra- mientas tecnológicas que esta exige para sus procesos, pero luego esta tecnología es prohibida como política interna de una de las partes?}

Aunque se trate de un supuesto poco probable, el arbitraje con el uso de las tecnologías se encuentra expuesto al hecho de que la tecnología pactada - directa o indirectamente, cuando las partes se someten a la institución y sus tecnologías - sea prohibida como política interna de una de las partes porque podría ponerse en riesgo información sensible de una parte, o de ambas. Si bien se trata de un acuerdo pactado voluntariamente por las partes, el tribunal arbitral deberá determinar qué resulta ser lo más conveniente sopesando el derecho de las partes a someter a arbitraje según lo acordado frente a la protección de datos.

Consideremos que en una relación jurídica determinada se establece acudir a una insti-

13. Decreto Legislativo 1071 — Ley de Arbitraje-, artículo 12, literal b).

14. Sin perjuicio de ello, cabe precisar que el artículo 35, numeral 2, de la Ley de Arbitraje establece que "(...) el tribunal arbitral podrá, previa consulta a las partes, reunirse en cualquier lugar que estime apropiado para oír a los testigos, a los peritos o a las partes, o para examinar o reconocer objetos, documentos o personas". Este extremo de la Ley de Arbitraje, para algunos, permitiría hacer audiencias en un lugar o espacio virtual.

15. Reglamento de Arbitraje de la Cámara de Comercio Internacional del 2017, artículo 3, numeral 2.

16. Reglamento de Arbitraje de la Cámara de Comercio Internacional del 2017, apéndice IV, literal f), Técnicas para la Conducción del Caso. 
tución arbitral y acogerse al uso de las tecnologías que esta institución proporciona para el desarrollo de las actuaciones arbitrales. No obstante, una de ellas, luego de hacer una evaluación exhaustiva respecto al uso de la tecnología " $x$ ", concluye que esta no es suficientemente segura y que pondría en riesgo absoluto la información de dicha entidad. Entonces, decide prohibir, como política interna, el uso de esta tecnología específica.

Esta cuestión quizás es una de las más delicadas porque entra en juego una decisión que resguarda los datos de la empresa - basada en la "ciberseguridad" en la mayoría de casosfrente a un acuerdo plenamente establecido por las partes dentro de un convenio arbitral.

La pregunta que podría surgirnos es, ¿no hubiera sido más diligente que, mientras no se haya determinado la política interna de uso de las tecnologías para los litigios, esta parte debía convenir otra solución como acudir a un arbitraje, pero sin el uso de dichas tecnologías en específico, o pactar un arbitraje que sea llevado únicamente de manera presencial, o acudir a otra institución arbitral que sea más flexible con el uso de las tecnologías? Imaginamos que sería un poco exigente cargar con ese riesgo - que es imputable únicamente a la empresa que establece la política interna de uso de tecnologías - a una parte que no ha generado esa situación.

No obstante, la solución a la que se arribe podría variar si es que, en el caso concreto, se determina que no solo se va a comprometer la seguridad de los datos de esa empresa sino también de la contraparte, por lo que en este caso lo más conveniente sería migrar a otra tecnología.

De igual manera, la respuesta no es tan sencilla y va a depender también el contexto que rodea a las partes. ¿Qué sucede si esta parte es el Estado? No sería exagerado si consideramos que en la mayoría de arbitrajes que se presentan en el Perú, una de las partes es el Estado. Entonces, si la política fuera prohibida por el Estado debido a que la tecnología pactada es potencialmente peligrosa y la decisión recae sobre todos los ministerios, organismos, órganos y demás instituciones públicas contratantes, ¿igual existiría una responsabilidad por parte de alguna entidad pública contratante?

¿Y si dos partes, cada una de distintos estados, han pactado determinada tecnología y, de un momento a otro, esa tecnología es prohibida por el estado de donde proviene una de ellas? No escaparía mucho de la realidad, si consideramos que existe una guerra comercial de plataformas virtuales -Zoom versus Google Meets o Facebook Rooms-, y que en China los ciudadanos se encuentran prácticamente imposibilitados de utilizar otras plataformas $y / 0$ programas que no sean las que brindan o permitan en ese país. ${ }^{17}$

Esta última interrogante más bien nos llevaría a concluir que por circunstancias ajenas a las partes la forma en que se llevaría a cabo el arbitraje debería modificarse, sea que las partes establezcan, de mutuo acuerdo, migrar a una nueva plataforma no prohibida en ningún estado, o que simplemente el arbitraje deba llevarse de manera presencial.

Las circunstancias deberán analizarse en cada caso concreto y por el tribunal arbitral. Así, si es que no existe un consenso, será el tribunal arbitral quien deberá evaluar qué es lo mejor para el caso concreto, tomando en cuenta si justificaría el uso de otra tecnología para no poner en riesgo los datos sensibles de cada parte.

De esa manera, que una parte prohíba justifica-

17. En China, la aplicación We Chat - lo que vendría a ser el "Facebook chino" — cuenta con varias funciones que engloba los servicios ofrecidos por Instagram, WhatsApp, Uber, Skype y hasta cuenta con un monedero virtual, que se conecta con las cuentas bancarias de los ciudadanos chinos. Mayor información en el siguiente enlace: https://rpp.pe/blog/geek-and-chick/ni-facebook-o-twitter-que-redes-sociales-usan-en-china-a-pesar-del-bloqueo-noticia-1079982?ref=rpp. 
damente como política interna, o le sea prohibida por un tercero, el uso de una determinada tecnología previamente pactada es un supuesto bastante remoto, pero igualmente delicado. No existe una respuesta concreta, y va a depender de la evaluación que realice el tribunal arbitral en el caso concreto.

\section{3. ¿Es más eficiente, en términos de costo y tiempo, establecer tecnologías para el ar- bitraje? Y de ser así, ¿es más eficiente esta- blecer las tecnologías a usar en el arbitraje antes o luego de iniciado el proceso?}

Resulta ser más eficiente, en términos de costo y tiempo, establecer tecnologías para el arbitraje; y se evita que gran parte de la discusión del caso se dedique a establecer qué tecnologías se usarán en el arbitraje, si es que las partes pactan estas tecnologías en el convenio arbitral y no en un momento posterior.

Cabe precisar que la cuestión planteada en esta sección está pensada únicamente respecto de la eficiencia o no, en términos de costo y tiempo, derivada de la implementación de tecnologías en el arbitraje.

Pues bien, la idea que podría rondarnos es que el uso de las tecnologías hace más eficiente al arbitraje. Y ello debido a que las tecnologías nos permiten ahorrar el costo asociado a la impresión, papel, copias, al personal y traslado del documento al lugar de presentación. Asimismo, se evita el tiempo que nos toma imprimir los documentos, ordenarlos, trasladarlos, entre otros.

Pero, ¿esto es cierto? Dependerá del momento $y$, definitivamente, del caso en concreto.

Como se mencionó en un principio, se recomienda que las partes establezcan detalladamente qué tipo de tecnologías se usarán en los arbitrajes al momento de celebrar el convenio arbitral. Con ello podrá evitarse que gran parte del arbitraje se enfoque en discutir qué tecnología es mejor para el arbitraje —-software, plataforma en la cual se cargarán los documentos, plataforma para audiencias, logística para las audiencias, etc.- y se pierda el enfoque principal: resolver la controversia.

Ahora, si nos encontramos en el supuesto en que las partes han pactado que el arbitraje se llevará mediante el uso de tecnologías pero no se ha establecido cuáles, definitivamente surgirá una discusión al momento de que las partes establezcan las reglas. Y en este caso puede ser que las propuestas de las partes no lleguen a conciliar.

Lo ideal es que las partes y el tribunal arbitral definan conjuntamente qué tecnología es la más eficiente para el caso concreto $-y$ para ello, evaluar factores como quiénes son las partes, quiénes conforman el tribunal arbitral, la materia del caso, la complejidad del mismo, etc.-, porque nadie más que los participantes del arbitraje sabrán qué es lo mejor para que se maneje correctamente el caso $^{18}$.

Definitivamente, si las partes no están de acuerdo en qué tecnología en específico se usará para las actuaciones arbitrales, la decisión deberá ser tomada por el tribunal arbitral. Y esta decisión podrá implicar que se use alguna de las propuestas ofrecidas por las partes, o ninguna de ellas acudiendo a una tercera establecida por el árbitro. Cabe precisar que el árbitro tomará una decisión basándose en qué resulta ser lo más eficiente para ambas partes y, en general, para el proceso.

Está claro que el tribunal arbitral no debe aceptar la propuesta de una de las partes que busca la aplicación de una tecnología en específico que genere que el arbitraje sea más oneroso para la otra parte.

Otro caso que podría presentarse $-y$ no esca-

18. Cámara de Comercio Internacional, Reporte de Tecnologías de Información en el Arbitraje Internacional (Francia, 2017), 7. 
pa nada de la realidad - es si las partes están tramitando el arbitraje de manera presencial y, por circunstancias ajenas, existe la necesidad inmediata de tramitarlo virtualmente. No pensemos tan lejos: lo que sucedió en nuestro país con los arbitrajes por las restricciones dictadas por el Gobierno del Perú, que comenzó a regir a partir del 16 de marzo de $2020^{19}$, por el crecimiento de contagios de la COVID-19.

Aquí los plazos de los arbitrajes quedaron suspendidos automáticamente -todos los centros de arbitraje suspendieron sus plazos porque el personal encargado de la administración ya no podía acudir a las oficinas de trabajo-. En esa línea, ilas partes debían ceder para que las actuaciones arbitrales se den exclusivamente de manera virtual?

La opción adoptada por algunos centros de arbitraje fue que, en la medida que sus reglamentos de arbitraje permitían las notificaciones por medios electrónicos y la posibilidad de realizar audiencias virtuales, y atendiendo a que en ese momento era imposible efectuar actuaciones presenciales, entonces puedan desarrollarse dichas actuaciones virtual y remotamente.

Ello supone una alternativa bastante interesante para que el arbitraje no se vea perjudicado y que se hizo viable dado que, en algún momento, hubo manifestación de las partes de ser notificadas vía correo electrónico.

Por lo tanto, en términos de costo y tiempo, es más eficiente establecer tecnologías, resultando más conveniente hacerlo antes - es decir, en el convenio arbitral — y no luego, en la medida que este último escenario supondrá que el arbitraje se extienda en exceso y se pierda la discusión principal: la solución de la controversia.

4. ¿Si se presentan problemas en el uso de las tecnologías, podrá dispensarse a las partes de utilizarlas?
En caso se presenten problemas en el uso de una tecnología determinada, el tribunal arbitral, evaluando el caso en concreto, tiene la facultad de dar instrucciones a las partes sobre el uso alternativo de una herramienta tecnológica para que sigan funcionando las actuaciones arbitrales virtuales.

Ello implica que a pesar de que sea una tecnología determinada pactada por las partes, el tribunal arbitral deberá velar por la eficiencia del arbitraje, y previa consulta a las partes, tomar la decisión sobre qué alternativa de herramienta tecnológica será la adecuada para el proceso.

Pero, ¿cambiaría el escenario si el problema estuviera relacionado a los costos en el uso de la tecnología convenida por las partes? Es decir, ¿qué sucede si las partes de un contrato han pactado el uso de una tecnología determinada que para una parte no supone un mayor costo - porque ya lo ha implementado en su uso diario-, mientras que para la otra parte implica un costo elevado por la adquisición del software y costo de implementación y aprendizaje?

En principio, podríamos considerar que esta parte no podría desconocer la tecnología que ha pactado en el convenio arbitral. Pero, ¿en todos los casos será así? La respuesta va a depender del caso en concreto en el que nos encontremos.

Por ejemplo, la decisión de continuar o no usando la tecnología, dependerá si el contrato es un contrato libremente firmado, donde ha habido una negociación ardua y extensa de las partes, o si nos encontramos frente a un contrato donde las partes no se hayan encontrado en igualdad para establecer las condiciones contractuales. Un claro ejemplo de esto último es el poco poder de negociación que existe en las contrataciones con el Estado, en donde el proveedor o contratista recibe un contrato modelo establecido por la entidad pública que debe ser

19. Medida dictada por el gobierno peruano a través del Decreto Supremo N 044-2020-PCM del 15 de marzo de 2020. 
firmado, de lo contrario recaería en responsabilidad por haber obtenido la buena pro y no haber firmado el contrato correspondiente.

Entonces, existen un sinfín de problemas que se pueden presentar - y se presentarán-con el uso de las tecnologías en el arbitraje. Una vez más, cada uno se irá resolviendo en cada caso concreto.

\section{5. ¿El tribunal arbitral puede establecer sanciones relacionadas a las actuaciones de las partes y el uso de tecnologías?}

Aunque todavía no sea una práctica común en la medida que es reciente la implementación obligatoria de las tecnologías en el arbitraje-, el tribunal arbitral tiene la facultad de sancionar a una de las partes si no está cooperando para que el arbitraje se encamine con el uso de las tecnologías.

Al ser el arbitraje un mecanismo de solución de conflictos que nace del consenso, se espera que las partes se desenvuelvan correctamente en el desarrollo del proceso.

Entonces, ¿qué sucede si una parte propone y exige constantemente que se apliquen unas herramientas tecnológicas que son bastante onerosas para el proceso, la contraparte e incluso para el tribunal arbitral? Si bien dependerá del caso concreto - porque podría darse que el caso sea tan complejo que amerite el uso de esa tecnología en específico-, consideremos que es una conducta que no se adecúa dentro de lo que se espera de las partes; por tanto, el tribunal arbitral podría sancionar a esta parte.

Otro escenario que podría suceder es que las partes hayan establecido en su convenio arbitral que las actuaciones arbitrales se realicen virtualmente, y luego, al momento de establecer las reglas, una de ellas sea reacia o formule una serie de objeciones que alarguen la decisión respecto de qué tecnología se va a implementar en el arbitraje. Esta conducta tampoco es adecuada para lo que se busca en un proceso arbitral.

Sea cual fuese el escenario, el tribunal arbitral tiene la posibilidad y facultad de sancionar a las partes debido a que una de ellas se está desenvolviendo de manera incorrecta, dado que, en los arbitrajes, las partes deben actuar de buena fe a lo largo del proceso y colaborar con el tribunal arbitral en el desarrollo del mismo. ${ }^{20}$

\section{Al momento de designar al árbitro, ¿la parte deberá tomar en cuenta si éste se encuentra familiarizado con el uso de las tecnologías pactadas?}

A fin de que un arbitraje sea llevado a cabo de manera eficiente, en términos de costo y tiempo, no solo las partes deben entender el uso de las tecnologías que han convenido, sino también los agentes que resolverán la controversia, por lo que es importante que la parte, dentro de las evaluaciones que realice sobre designar o no a un árbitro, verifique que éste al menos tenga una noción básica del funcionamiento de la tecnología pactada.

Para ello, es importante que, previamente a la designación del árbitro, la parte consulte con el posible árbitro a designar si éste se encuentra familiarizado con las herramientas tecnológicas convenidas. ${ }^{21}$

En este nuevo contexto surge la duda de si un árbitro, además de revelar sobre la imparcialidad e independencia, debe declarar si se encuentra familiarizado con el manejo del arbitraje con las

20. Artículo 38 de la Ley de Arbitraje: "Las partes están obligadas a observar el principio de buena fe en todos sus actos e intervenciones en el curso de las actuaciones arbitrales y a colaborar con el tribunal arbitral en el desarrollo del arbitraje."

21. Cámara de Comercio Internacional, Reporte de Tecnologías de Información en el Arbitraje Internacional (Francia, 2017), 6. 
tecnologías convenidas por las partes. Al respecto, no hay una exigencia legal que obligue a los árbitros a revelar en el arbitraje si se encuentra familiarizado o no con una herramienta tecnológica en específico, en la medida que la obligación recae únicamente en la revelación de circunstancias que pudieran generar dudas sobre la imparcialidad e independencia de un árbitro ${ }^{22}$.

Tampoco una parte podría recusar a un árbitro por no conocer o estar familiarizado con la herramienta tecnológica pactada en el convenio arbitral, dado que no se trata de una cualidad del árbitro.

Sin perjuicio de ello, podría darse el caso que las partes expresamente establezcan en el convenio arbitral que el árbitro a designar deba conocer sobre la herramienta tecnológica pactada. Únicamente en este escenario, al tratarse de una exigencia convenida por las partes sobre una cualidad con la que debe contar el árbitro, cualquiera de las partes tendría la posibilidad de cuestionar la designación de un árbitro si es que éste no se encuentra familiarizado con las tecnologías pactada por las partes ${ }^{23}$.

Por lo tanto, cualquiera de las partes, al momento de designar a un árbitro, debe considerar que éste maneje, al menos en lo básico, la tecnología pactada para que el arbitraje sea más eficiente.

7. ¿Se ha generado un nuevo derecho de las partes en el arbitraje de "testear" la conectividad, audio y video antes de las audiencias? ¿Qué sucede si se le niega esta solicitud a una parte?
Otra cuestión que surge es si las partes pueden solicitar al tribunal arbitral, previamente a una audiencia, que exista una prueba - "testeo"de su conectividad, o del uso de su micrófono o cámara de video antes de llevar a cabo una audiencia, con la finalidad de que esa parte se cerciore que cuenta con los requisitos técnicos mínimos para que pueda desenvolverse correctamente en una audiencia y que el tribunal arbitral pueda entender con claridad su posición.

¿El tribunal está obligado a acceder a este pedido o podría dispensar de ello y directamente realizar la audiencia? Es una cuestión que resuena en el supuesto que las partes hayan pactado el uso de las tecnologías y audiencias virtuales.

Ello, porque estamos frente a un pedido que implica que la parte se sienta con la seguridad de que su derecho de defensa no se va a ver mermado por un tema técnico y que puede ser solucionado detectándose a tiempo.

Por ejemplo, el Protocolo de Seúl exige, dentro de las buenas prácticas que deben existir para la videoconferencia o audiencia virtuales, que previamente a la videoconferencia se permita a las partes tener dos pruebas: una, antes de la audiencia; y otra, inmediatamente antes de la videoconferencia ${ }^{24}$, con la finalidad que el tribunal arbitral se cerciore de que no existe ningún problema para que las partes -o las pruebas que se actuarán, como una pericia o una declaración - hagan valer sus derechos. La misma recomendación del testing previo a la audiencia es brindada en el Reporte $\mathrm{CCl}^{25}$

22. Artículo 28, numeral 1 de la Ley de Arbitraje: "Todo árbitro debe ser y permanecer, durante el arbitraje, independiente e imparcial. La persona propuesta para ser árbitro deberá revelar todas las circunstancias que puedan dar lugar a dudas justificadas sobre su imparcialidad e independencia."

23. Artículo 28, numeral 3 de la Ley de Arbitraje: "Un árbitro solo podrá ser recusado si concurren en él, circunstancias que den lugar a dudas justificadas sobre su imparcialidad o independencia, así como no poseer las calificaciones convenidas por las partes o las establecidas por el reglamento de la institución arbitral o las exigidas por la ley."

24. Artículo 6 del Protocolo de Seúl.

25. Cámara de Comercio Internacional, Reporte de Tecnologías de Información en el Arbitraje Internacional (Francia, 2017), 16. 
Aunque parezca un poco exagerado, ante la negativa del tribunal arbitral de realizar una reunión de prueba para verificar que las partes no tienen ningún inconveniente con el cumplimiento de los requisitos técnicos mínimos, la parte podría activar su derecho a acudir a la instancia judicial en vía de anulación por no poder hacer valer sus derechos. ${ }^{26}$ Efectivamente, el tribunal arbitral tiene que asegurar que el uso de las tecnologías no interfiera con el tratamiento igualitario de las partes. ${ }^{27}$

Por lo tanto, el uso de las tecnologías en el arbitraje ha "generado" un derecho de las partes de cerciorarse que cumplen con los requerimientos técnicos mínimos para que su posición pueda transmitirse al tribunal arbitral sin inconvenientes.

\section{CONCLUSIÓN}

El arbitraje ha ido adaptándose a las nuevas circunstancias. Primero, ha ido flexibilizándose, separándose de la manera rigurosa de cómo el poder judicial maneja sus procesos.

Luego, continuando con el proceso evolutivo, el arbitraje ha logrado incorporar figuras como la confirmación de árbitros, el arbitraje de emergencia, las Reglas de la IBA, las Reglas de Praga, el arbitraje acelerado, entre otros.

El arbitraje ha tenido que "remasterizarse", principalmente por la llegada de la pandemia de enfermedad originada por la COVID-19, teniendo que implementar, como uso obligatorio, herramientas de la tecnología. De esa manera, la tecnología pasó de ser la excepción a ser la regla general en un arbitraje.

La implementación de la tecnología en las actuaciones arbitrales supone que se genere una serie de situaciones y cuestiones que son nuevas y a las cuales los usuarios del arbitraje —abogados, árbitros, partes del litigio, secretarios arbitrales, etc.- se van a tener que enfrentar en adelante. En este trabajo se ha tratado de revisar algunos — de las muchos-incidentes que pudieran presentarse.

Es importante recordar que las partes son pieza clave para que las tecnologías puedan implementarse y desarrollarse sin inconvenientes, y el tribunal arbitral será, como siempre, quien tenga la última decisión sobre qué es lo más conveniente para que las tecnologías funcionen en un arbitraje y puedan superarse los incidentes que vayan apareciendo en el camino. Al final, es un costo que debemos asumir por la "remasterización" del arbitraje.

26. Artículo 63, numeral 1, inciso b) de la Ley de Arbitraje.

27. Cámara de Comercio Internacional, Reporte de Tecnologías de Información en el Arbitraje Internacional (Francia, 2017), 7. 